\title{
Defect Microstructural Equivalence in Molybdenum Under Different Irradiation Conditions At
}

\section{Low Temperatures and Low Doses}

Donghua $\mathrm{Xu}^{1 *}$, Gerrit VanCoevering ${ }^{2}$, Brian D. Wirth ${ }^{1}$

${ }^{1}$ Department of Nuclear Engineering, University of Tennessee, Knoxville, TN 37996, USA

${ }^{2}$ Department of Nuclear Engineering and Radiological Sciences, University of Michigan, Ann Arbor, MI 48109, USA

\begin{abstract}
Owing to its advantages such as higher dose rates and shorter time required, ion irradiation, including in-situ ion irradiation of nano-foils on electron microscopes, is being proposed as a surrogate for neutron/reactor irradiation in materials testing for the research of irradiation induced/enhanced degradation of reactor structural materials. This raises a key question: can the microstructural evolution be matched across different irradiation conditions? Here we report our recent study using a rate-theory based cluster dynamics approach on the time dependent defect accumulation in elemental molybdenum under varying ion and neutron irradiation conditions, within a low temperature and low dose regime. The approach solves a large system of coupled differential equations each equation describing the rate of change in the concentration of a particular defect/cluster (defined by its nature and number of constituent point defects) caused by direct production (for primary defects), diffusion flux across spatial grids (for mobile defects, under ion irradiation), and capturing and/or emission interactions with other defects/clusters. Our results suggest that defect evolution under different dose rates can be very well matched in either thin foil ion irradiation or bulk neutron irradiation, by using different irradiation temperatures to counteract the effect of the dose rate variation. Across the two types of irradiation, less perfect matching is obtained mainly due to the strong surface sink effect in the thin foil, but the matching can be improved by combining the temperature shift strategy with selection of a single depth in the foil (as opposed to all depths averaged) for matching, or a greater foil thickness.
\end{abstract}

(C) 2015. This manuscript version is made available under the Elsevier user license http://www.elsevier.com/open-access/userlicense/1.0/ 
Key Words: microstructural evolution, radiation defects, radiation effects, ion irradiation, neutron irradiation

*Corresponding Author, Tel. (865)245-9861; Fax: (865)974-0668; Email: xudh@utk.edu.

\section{Introduction}

With an exceptionally high energy density and low greenhouse gas emission, nuclear power has regained interest as the world strives for sustainability in energy and environment. New generation nuclear power reactors are being designed while those having operated for more than 30 years are being considered for lifetime extension. Neutron irradiation in a nuclear power reactor is known to cause significant property degradation (e.g., embrittlement and hardening) of structural materials, which represents a major safety concern in both the new reactor design and the lifetime extension of existing reactors. ${ }^{1-6}$ A significant amount of cross-disciplinary research is being conducted in the United States and globally with the goal to predict the degree of materials degradation as a function of extended service time or irradiation dose (totaling $>100 \mathrm{dpa}) .^{3-8}$ To reach the target dose, neutron irradiation of sample materials in a test reactor takes at minimum a few years due to its relatively low dose rate (3-8 dpa/yr for thermal test reactors and $20 \mathrm{dpa} / \mathrm{yr}$ for fast spectrum test reactors ${ }^{4}$ ), which is not so much higher than that in a power reactor (2-3 dpa/yr for pressurized water reactors $\left.{ }^{3}\right)$ as would be desired for predictive studies. Characterization of test-reactor irradiated sample materials requires special precautions and dedicated facilities because of induced sample radioactivity. In contrast, ion irradiation can be performed at a dose rate several orders of magnitude above that in a power reactor or a test reactor, and thus requires much shorter time than test-reactor neutron irradiation in order to reach the same target dose level in the sample materials. Ion irradiation normally does not induce sample radioactivity, and hence characterization of ion-irradiated materials requires no dedicated facilities. Therefore, ion irradiation has been proposed as a surrogate for neutron/reactor irradiation in the study of irradiation induced/enhanced degradation of reactor structural materials, with some supportive experimental evidence reported recently. ${ }^{8}$ 
For a given material, the property degradation under irradiation is caused and determined primarily by the microstructural evolution driven by the continuous production and kinetic diffusion and interactions of lattice defects (e.g., vacancies, self-interstitials, and their respective clusters). Hence, the degree to which the property degradation measured under one irradiation condition represents that occurring under another irradiation condition is determined by how closely the microstructural evolution, essentially the defect evolution, can be matched across the different irradiation conditions. Ion irradiation is known to differ from neutron/reactor irradiation in multiple aspects, including dose rate and probabilistic and spatial distributions of primary defects (those generated directly during collision cascades, prior to long range diffusion and interactions). In the case of in-situ TEM (Transmission Electron Microscopy) ion irradiation, an advanced experimental technique that allows real-time observation of microstructural/defect evolution during simultaneous ion irradiation, ${ }^{9-10}$ the small (tens to a few hundred nanometer) thickness of a TEM foil and the related surface sink effect on mobile defects represent another key aspect distinct from neutron irradiation. Given these major differences, a significant question arises: can the microstructural/defect evolution be matched across different irradiation conditions?

While fully addressing this question will require long time effort of the research community, we report in this paper some interesting results from our recent investigation. Here we focus on low temperature low dose ion irradiation in thin molybdenum (Mo) foils and neutron irradiation in bulk Mo, and explore the possibility of using a shift in irradiation temperature to match the microstructural/defect evolution path as the dose rate changes in either type of irradiation, or upon switching between the two types of irradiation. Mo is chosen as an example because of the availability of a relatively well calibrated and tested set of defect related data/parameters.

It should be noted that the application of a temperature shift was originally proposed by Mansur to obtain invariance in the steady-state swelling rate or the steady-state rate of point defects being absorbed by sinks upon changes in the dose rate. ${ }^{11-12}$ For those purposes Mansur derived several analytical expressions of required temperature shifts, based on steady-state solutions to point defect (only) rate equations with the following assumptions/simplifications: 1 . only point defects are directly generated by 
collision cascades; 2. only point defects are mobile; 3 . defect cluster concentrations are not evolving; 4. there are no free surfaces. Here, we are concerned with the temporal evolution of concentrations of all defects (point defects and their clusters) and corresponding size distribution, rather than the steady state rates of particular processes. As detailed below, we also remove the assumptions/simplifications needed in the analytical derivations by Mansur.

\section{Results and Discussion}

\section{$\underline{\text { Ion irradiation at varying dose rates }}$}

We first examine low temperature $1 \mathrm{MeV} \mathrm{Kr}$ ion irradiation in a $60 \mathrm{~nm}$ thick Mo foil at two different fluxes, $1.6 \times 10^{9}$ and $1.6 \times 10^{11}$ ions $\mathrm{cm}^{-2} \mathrm{~s}^{-1}$, which correspond to nominal dose rates of $3 \times 10^{-6}$ and $3 \times 10^{-4} \mathrm{dpa} \mathrm{s}^{-1}$ (dpa: displacements per atom), respectively, as calculated by SRIM-2008 ${ }^{13}$ in the "quick calculation" mode with a displacement energy of $60 \mathrm{eV}$ and then averaged over the foil thickness (thus neglecting the weak depth dependence of damage production predicted by SRIM). SRIM indicates that most ions penetrate through the foil, and hence only intrinsic point defects (vacancies $V$ and selfinterstitials $I)$ and their clusters $\left(V_{m}, I_{n}\right)$ are considered here. During their travel inside the foil, the ions directly produce certain small defects, i.e., the primary defects, through collision cascades. Some defects (primary or later formed) are mobile and undergo long-range thermal diffusion. The mobile defects can be captured by other mobile or immobile defects that they encounter during migration. In addition, defect clusters may emit point defects due to thermally activated dissociation. Incorporating all these fundamental processes, the defect evolution can be described by a system of coupled differential equations with the following generic form:

$$
\left\{\frac{\partial C_{c l s}^{x_{i}}}{\partial t}=\times P_{c l s}^{x_{i}}+D_{c l s} \frac{\partial^{2} C_{c l s}^{x_{i}}}{\partial x^{2}}+G R T+G R E \quad A R T \quad A R E \quad S R\right\}
$$

where $C_{c l s}^{x_{i}}$ is the volumetric concentration $\left(\right.$ in $\mathrm{nm}^{-3}$ ) of a defect/cluster $(c l s)$ at the $i$-th depth grid $\left(x_{i}\right.$, in $\mathrm{nm}$ ) below the implanted surface, $\varphi$ is the incident particle (here ion) flux (in ion $\mathrm{nm}^{-2} \mathrm{~s}^{-1}$ ), $P$ is the 
probability (in ion ${ }^{-1} \mathrm{~nm}^{-1}$ ) of direct generation by collision cascades, $D$ is the diffusivity, $G R T$ is the rate of generation of $c l s$ by trapping reactions $(A+B \rightarrow c l s)$ among other defects/clusters, GRE is the rate of generation of $c l s$ by emission processes $(A \rightarrow c l s+B)$ of other clusters, $A R T$ is the rate of annihilation of $c l s$ by its trapping reaction with other clusters $(c l s+A \rightarrow B), A R E$ is the rate of annihilation of $c l s$ by its own emission process ( $c l s \rightarrow A+B$ ), and $S R$ is the rate of $c l s$ sinking to pre-existing line dislocations (note that we consider coarse grained molybdenum and hence neglect grain boundary effect as a potential sink of defects; our experimental reference ${ }^{21}$ had a Mo grain size of $\left.\sim 70 \mu \mathrm{m}\right)$.

More exact forms of the equations for different defects/clusters are as follows:

$$
\begin{aligned}
& \frac{\partial C_{V_{1}}^{x_{i}}}{\partial t}=\times P_{V_{1}}^{x_{i}}+D_{V_{1}} \frac{\partial^{2} C_{V_{1}}^{x_{i}}}{\partial x^{2}}+\sum_{n>0} k_{I_{n}, V_{n+1}}^{+} C_{I_{n}}^{x_{i}} C_{V_{n+1}}^{x_{i}}+\left(\sum_{n>2} k_{V_{n}} C_{V_{n}}^{x_{i}}+2 k_{V_{2}} C_{V_{2}}^{x_{i}}\right) \\
& \left(\sum_{n>0} k_{I_{n}, V_{1}}^{+} C_{I_{n}}^{x_{i}} C_{V_{1}}^{x_{i}}+\sum_{n>1} k_{V_{n}, V_{1}}^{+} C_{V_{n}}^{x_{i}} C_{V_{1}}^{x_{i}}+2 k_{V_{1}, V_{1}}^{+}\left(C_{V_{1}}^{x_{i}}\right)^{2}\right) \quad{ }_{\text {disl }} D_{V_{1}} C_{V_{1}}
\end{aligned}
$$

for the single vacancy,

$$
\left\{\begin{aligned}
\frac{\partial C_{V_{m}}^{x_{i}}}{\partial t}=\times & P_{V_{m}}^{x_{i}}+D_{V_{m}} \frac{\partial^{2} C_{V_{m}}^{x_{i}}}{\partial x^{2}}+\left(\sum_{n>0} k_{I_{n}, V_{n+m}}^{+} C_{I_{n}}^{x_{i}} C_{V_{n+m}}^{x_{i}}+\sum_{m>n>0} k_{V_{n}, V_{m}}^{+} C_{V_{n}}^{x_{i}} C_{V_{m}}^{x_{i}}\right)+k_{V_{m+1}} C_{V_{m+1}}^{x_{i}} \\
& \left(\sum_{n>0} k_{I_{n}, V_{m}}^{+} C_{I_{n}}^{x_{i}} C_{V_{m}}^{x_{i}}+\sum_{n>0} k_{V_{n}, V_{m}}^{+} C_{V_{n}}^{x_{i}} C_{V_{m}}^{x_{i}}+k_{V_{m}, V_{m}}^{+}\left(C_{V_{m}}^{x_{i}}\right)^{2}\right) k_{V_{m}} C_{V_{m}}^{x_{i}} \quad{ }_{d i s l} D_{V_{m}} C_{V_{m}}
\end{aligned}\right\}
$$

for vacancy clusters $V_{m}(m>1)$,

$$
\begin{aligned}
\frac{\partial C_{I_{1}}^{x_{i}}}{\partial t}= & \times P_{I_{1}}^{x_{i}}+D_{I_{1}} \frac{\partial^{2} C_{I_{1}}^{x_{i}}}{\partial x^{2}}+\sum_{n>0} k_{V_{n}, I_{n+1}}^{+} C_{V_{n}}^{x_{i}} C_{I_{n+1}}^{x_{i}}+\left(\sum_{n>2} k_{I_{n}} C_{I_{n}}^{x_{i}}+2 k_{I_{2}} C_{I_{2}}^{x_{i}}\right) \\
& \left(\sum_{n>0} k_{V_{n}, I_{1}}^{+} C_{V_{n}}^{x_{i}} C_{I_{1}}^{x_{i}}+\sum_{n>1} k_{I_{n}, I_{1}}^{+} C_{I_{n}}^{x_{i}} C_{I_{1}}^{x_{i}}+2 k_{I_{1}, I_{1}}^{+}\left(C_{I_{1}}^{x_{i}}\right)^{2}\right) z_{\text {disl }} D_{I_{1}} C_{I_{1}}
\end{aligned}
$$

for the single interstitial, and

$$
\left\{\begin{aligned}
\frac{\partial C_{I_{m}}^{x_{i}}}{\partial t}= & \times P_{I_{m}}^{x_{i}}+D_{I_{m}} \frac{\partial^{2} C_{I_{m}}^{x_{i}}}{\partial x^{2}}+\left(\sum_{n>0} k_{V_{n}, I_{n+m}}^{+} C_{V_{n}}^{x_{i}} C_{I_{n+m}}^{x_{i}}+\sum_{m>n>0} k_{I_{n}, I_{m n}}^{+} C_{I_{n}}^{x_{i}} C_{I_{m n}}^{x_{i}}\right)+k_{I_{m+1}} C_{I_{m+1}}^{x_{i}} \\
& \left(\sum_{n>0} k_{V_{n}, I_{m}}^{+} C_{V_{n}}^{x_{i}} C_{I_{m}}^{x_{i}}+\sum_{n>0} k_{I_{n}, I_{m}}^{+} C_{I_{n}}^{x_{i}} C_{I_{m}}^{x_{i}}+k_{I_{m}, I_{m}}^{+}\left(C_{I_{m}}^{x_{i}}\right)^{2}\right) k_{I_{m}} C_{I_{m}}^{x_{i}} z_{\text {disl }} D_{I_{m}} C_{I_{m}}
\end{aligned}\right\}
$$

for interstitial clusters $I_{m}(m>1)$, where $k^{+}$is the capturing rate constant (in $\mathrm{nm}^{3} \mathrm{~s}^{-1}$ ), $k$ is the emission rate constant (in $\mathrm{s}^{-1}$ ), $z$ is the interstitial-dislocation bias (dimensionless, also applied to interstitial- 
interstitial type capturing/emission rate constants) and $\rho$ is the pre-existing dislocation line density (in $\mathrm{nm}^{-}$ ${ }^{2}$ ). These equations can be further simplified for those defects not directly generated by irradiation and those that are not mobile. We have preciously developed a high efficiency FORTRAN code named PARASPACE (short for PARAllel SPAtially-dependent Cluster Evolution) to solve such an equation system, and the details about the code (design, structure, algorithms, leading features etc.) can be found in Reference 14. The capturing and emission rate constants are determined by the diffusivities and binding energies $\left(E^{B}\right)$ of the relevant defects as: $k_{m, l}^{+}=4 \quad\left(r_{m}+r_{l}\right) \quad\left(D_{m}+D_{l}\right)$ (for true or effective 3D diffusionreaction systems, derived by Waite [15]) and $k_{l}=k_{l 1,1}^{+} C_{0} \exp \left(\frac{E_{l}^{B}}{k_{B} T}\right)$, where $r$ is the defect radius and $C_{0}$ is the atomic number density of the matrix. One may argue that the above capturing rate constant expression should be reduced by a factor of two, when applied to capturing processes involving two identical species (e.g. $\mathrm{V}_{1}+\mathrm{V}_{1} \rightarrow \mathrm{V}_{2}$ ), due to indistinguishability. This has not been clearly discussed in either Waite's original publication or publications in the field of radiation damage utilizing the expression (e.g. [16]). Here we point out that at least for all the calculations in this study, the influence of having or not having the factor-of-2 reduction for identical-species capturing events is negligible. It is also noted that some previous studies chose to use expressions that differ significantly from Waite's formula. For example, in [17], $4 \boldsymbol{r}_{V_{1}} \boldsymbol{D}_{V_{1}}$ was used to describe the $\mathrm{V}_{1}+\mathrm{V}_{1} \rightarrow \mathrm{V}_{2}$ reaction. The inconsistency in the literature on this point may be worth examining in the future, but is beyond the scope of the present study.

On the two free surfaces of the foil, perfect sink boundary conditions are applied, in other words, all the defects are set to zero concentration except single point defects which are set to respective thermal equilibrium concentrations $C_{0} \exp \left(\frac{E_{I \text { or } V}^{F}}{k_{B} T}\right)$ (very small at low temperatures), where $E^{F}$ is the formation energy ( $3 \mathrm{eV}$ for $V, 7.4 \mathrm{eV}$ for $I$, according to previous ab initio calculations in $\mathrm{Mo}^{18}$ ).

The identities and production probabilities (per ion per unit length) of primary defects used in this work are listed in Table S1 (supplementary material ${ }^{19}$ ). These are based on the recent molecular dynamics (MD) simulations ${ }^{20}$ of collision cascades in Mo, which revealed the direct production of not only point 
defects but also defect clusters by PKAs (primary knock-on atoms) with an energy above $1 \mathrm{keV}$. The MD simulations also provided the survival efficiency of point defects and their probabilities of forming different sized clusters for different PKA energies, which were combined with the SRIM calculated PKA energies and populations, following a procedure elaborated in Reference 14, to yield the production probabilities listed in Table S1. Table S2 (supplementary material ${ }^{19}$ ) lists the mobile species and their diffusivity parameters used in this work, which were calibrated and tested against a fairly large volume of data from low dose TEM in-situ ion $(1 \mathrm{MeV} \mathrm{Kr})$ irradiation experiments in $\mathrm{Mo}$ at $80{ }^{\circ} \mathrm{C} .{ }^{21}$ It is noted that the data listed in Tables S1 and S2 are somewhat different from those included in Reference 14 because of the adoption of the new information from the recent MD cascade simulations specifically for Mo. Comparisons of model predictions using the updated parameters with the $80{ }^{\circ} \mathrm{C} \mathrm{Kr}$ ion irradiation experiments are provided in Figs. S1 and S2 (supplementary material ${ }^{19}$ ). The main purpose of the present paper, however, is not to find the accurate defect production and mobility data. For the cluster binding energies, expressions based on the standard capillary law are used, specifically, $\left.7.410 .56 \times\left[\begin{array}{lll}n^{2 / 3} & (n & 1\end{array}\right)^{2 / 3}\right]$ for interstitial clusters and $34.56 \times\left[\begin{array}{lll}n^{2 / 3} & \left(\begin{array}{ll}n & 1\end{array}\right)^{2 / 3}\end{array}\right]$ for vacancy clusters.

Figure 1(a) shows the defect concentration evolution in the $60 \mathrm{~nm}$ Mo foil at the aforementioned two dose rates that differ by two orders of magnitude, but at a common temperature of $80{ }^{\circ} \mathrm{C}$. At each dose rate, the defect evolution is represented by three quantities, namely, the total (isolated and clustered) vacancy concentration, the total interstitial concentration, and the TEM visible (diameter $>1.5 \mathrm{~nm}$, extinction due to imaging conditions not considered here) interstitial loop number density, each as a function of dose. These quantities were averaged over the foil thickness to reflect the overall defect evolution within the foil. As can be seen in Fig. 1(a), these concentrations/densities all increase with dose, although the loop density exhibits a clear trend of saturation within the dose range explored. At each dose rate, the total vacancy concentration is noticeably higher than the total interstitial concentration, which is because single interstitials and small interstitial clusters are highly mobile and consequently a considerable fraction of total interstitials diffuse to and annihilate at the free surface sinks. However, the 
difference between the orders of magnitude of the total vacancy and total interstitial concentrations tends to diminish as the dose increases, because of the "shielding" effect of gradually formed interstitial loops which capture the small mobile interstitial species and reduce their sinking to the free surfaces. When the dose rate changes from $3 \times 10^{-6}$ to $3 \times 10^{-4} \mathrm{dpa} \mathrm{s}^{-1}$, the time available for the mobile interstitial species from the interior of the foil to reach the free surfaces is reduced and hence a higher total interstitial concentration is built up from the very beginning of the irradiation, while the total vacancy concentration is initially not affected. Subsequently, as the irradiation continues, the higher interstitial concentration effectively enhances interstitial-interstitial clustering relative to interstitial-vacancy recombination since the former involves two interstitial species, although both processes are second order reactions and both subject to the shorter timeframe at the higher dose rate. This then results in a higher interstitial loop density as well as a higher total vacancy concentration at the higher dose rate.

Figure 1(b) shows that this dose rate effect on the defect concentration evolution in the ion irradiated thin foil can be well compensated by a single temperature shift. Using a higher temperature, $158{ }^{\circ} \mathrm{C}$ (determined through manual fitting of the concentration/density curves, with an estimated error of $\pm 1.5^{\circ} \mathrm{C}$ ) at the $3 \times 10^{-4} \mathrm{dpa} \mathrm{s}^{-1}$ dose rate, as opposed to the $80{ }^{\circ} \mathrm{C}$ at the $3 \times 10^{-6} \mathrm{dpa} \mathrm{s}^{-1}$, nearly identical defect evolution paths are obtained, despite the two orders of magnitude difference between the two dose rates. Figure 2 compares the size distribution curves (averaged over the foil thickness) of interstitial clusters (main figure) and vacancy clusters (inset) for these two irradiation conditions (same as in Fig. 1(b)) at a common dose of 1 dpa. Size distribution curves were also examined at other doses (not plotted, for clarity purpose), which showed similar agreement between the two irradiation conditions to that at 1 dpa. Evidently, the temperature shift in this case not only counteracts the effect of the changing dose rate on the defect concentration/density, but also produces nearly the same size distribution of defect clusters at the two significantly different dose rates. It is worth noting that the non-uniform defect evolution at different depth positions may cause some spurious shoulders/peaks on the depth averaged size distribution curve if the spatial mesh used in the modeling is not fine enough, as shown in Fig. S3 
(supplementary material $^{19}$ ). A fine spatial mesh with a spacing of $1.5 \mathrm{~nm}$ was used to suppress the spurious features and obtain the truthful distribution curves in Fig. 2. By contrast, the depth averaged defect densities are less sensitive to the spatial mesh.

While it is expected that increasing the temperature can accelerate the defect sinking to free surfaces and defect-defect interaction and recombination, the degree of matching across the two different irradiation conditions is quite surprising, considering the many pieces of physical details involved in the problem, e.g., multiple mobile species with different mobilities, multiple primary defects, intricate competition among multiple fundamental processes and the time dependence of concentrations of all defects.

\section{$\underline{\text { Neutron irradiation at varying dose rates }}$}

Next we examine defect evolution in bulk Mo under neutron relevant irradiation conditions. The same system of rate equations and the same defect kinetic and energetic parameters as above are used except with two modifications to the equations: 1). removing the spatial dependence, and 2). changing the cascade production probabilities and the particle flux to values representing the neutron conditions. The first modification is because the free surfaces are no longer the dominant sink and the primary defects are produced homogeneously by neutrons. For the cascade production probabilities (per neutron per unit length), the PKA energy distribution and population calculated by the SPECTER ${ }^{22}$ code that correspond to an actual neutron spectrum at a specific position inside the High Flux Isotope Reactor (HFIR) at the Oak Ridge National Laboratory were combined with the aforementioned MD cascade simulation results, yielding the probability values listed in Table S3 (supplementary material ${ }^{19}$ ).

The lines in Fig. 3(a) represent the defect evolution at $80{ }^{\circ} \mathrm{C}$ in the HFIR neutron irradiated Mo at an actual HFIR neutron flux, $8 \times 10^{14} \mathrm{n} \mathrm{cm}^{-2} \mathrm{~s}^{-1}(>0.1 \mathrm{MeV})$, corresponding to a nominal dose rate of $4.5 \times 10^{-7} \mathrm{dpa} / \mathrm{s}$ in Mo according to SPECTER. The dose dependence of the three plotted concentrations/densities is similar to that in the thin foil ion irradiation case. A significant difference, however, is that in the neutron case, the total vacancy and total interstitial concentration trajectories 
overlap each other. This is because the dominant defect annihilation mechanism is recombination here which reduces interstitial and vacancy populations equally, as opposed to the surface sink mechanism in the thin foil ion irradiation case, which preferentially reduces the more mobile interstitial defects. It should be noted that a low pre-existing dislocation line density of $10^{10} \mathrm{~m}^{-2}$ is used throughout this study, to be consistent with the related Mo experiments, ${ }^{21,23}$ and correspondingly, the dislocation sink mechanism has little influence. Also plotted in Fig. 3(a) are the results (symbols) for an artificially increased HFIR neutron flux, $8 \times 10^{17} \mathrm{n} \mathrm{cm}^{-2} \mathrm{~s}^{-1}(>0.1 \mathrm{MeV})$, equivalent to a nominal dose rate of $4.5 \times 10^{-4}$ dpa/s, still at $80{ }^{\circ} \mathrm{C}$. The higher dose rate shortens the time available for recombination, and hence leads to higher total interstitial and total vacancy concentrations from the beginning of the irradiation. Although both types of point defects have higher total concentrations, the interstitial-interstitial clustering is favored next over interstitial-vacancy recombination due to the bias (1.2 here, selected near the range of values used in previous studies ${ }^{16,24}$ ) and the greater rate constants (involving two interstitial defect diffusivities) of the former process. This results in a higher interstitial loop density as well as more preserved (but still balanced) total interstitial and total vacancy concentrations at increasing doses.

Figure 3(b) shows that by selecting a higher temperature, $217{ }^{\circ} \mathrm{C}$, the defect concentration evolution in the neutron irradiated bulk Mo at the higher dose rate of $4.5 \times 10^{-4} \mathrm{dpa} / \mathrm{s}$ can be matched really well to that at the lower dose rate, $4.5 \times 10^{-7} \mathrm{dpa} / \mathrm{s}$, and the lower temperature, $80{ }^{\circ} \mathrm{C}$. Figure 4 shows the defect size distribution curves at $1 \mathrm{dpa}$ for these two irradiation conditions (same as in Fig. 3(b)). Compared with the condition of $4.5 \times 10^{-7} \mathrm{dpa} / \mathrm{s}$ and $80{ }^{\circ} \mathrm{C}$, the size distribution of the $4.5 \times 10^{-4} \mathrm{dpa} / \mathrm{s}$ and $217^{\circ} \mathrm{C}$ irradiation is only slightly shifted towards larger sizes, with the peak for interstitial clusters moved from $\sim 9 \mathrm{~nm}$ to $\sim 9.5 \mathrm{~nm}$, for example. The mean interstitial cluster radius and mean vacancy cluster radius are $8.1 \mathrm{~nm}$ and $0.17 \mathrm{~nm}$, respectively, for the lower dose rate and lower temperature irradiation, and 8.6 $\mathrm{nm}$ and $0.18 \mathrm{~nm}$ for the higher dose rate and higher temperature irradiation. Overall, the size distributions of the two irradiation conditions are in excellent agreement. Like in the cases of varying ion irradiation conditions in the previous section, the ability of a single temperature shift here to counteract the neutron 
dose rate effects on the defect concentration/density and size distribution is quite surprising, given the complexity of the defect evolution kinetics as mentioned earlier.

\section{$\underline{\text { Ion irradiation vs. neutron irradiation }}$}

Finally, we examine the possibility of matching defect evolution across bulk neutron irradiation and thin foil ion irradiation using a single temperature shift. We fix the neutron irradiation to the actual HFIR reactor condition $\left(4.5 \times 10^{-7} \mathrm{dpa} / \mathrm{s}, 80^{\circ} \mathrm{C}\right)$ and the $\mathrm{Kr}$ ion irradiation dose rate to the $3 \times 10^{-4} \mathrm{dpa} \mathrm{s}^{-1}$, as mentioned above, and only adjust the ion irradiation temperature. The best matching we obtained this way is shown in Fig. 5, with an ion irradiation temperature of $190{ }^{\circ} \mathrm{C}$. While the interstitial loop density can be matched quite well once the dose reaches 0.001 dpa and above, the total vacancy and total interstitial concentration trajectories of the thin foil ion irradiation fall below and above those of the bulk neutron irradiation, respectively. This is a result of the strong surface sink effect in the $60 \mathrm{~nm}$ thin foil as discussed earlier. As shown in Fig. 6, the size distribution curves for these two irradiation conditions exhibit fair agreement (although not as good as in Figs. 2 and 4), with some shift $(\sim 2 \mathrm{~nm})$ in the peak position of interstitial clusters. Note that the size distribution here in Fig. 6 for the thin foil ion irradiation is an average over the foil thickness, and that a relatively dense mesh of depth positions with a spacing of $1.5 \mathrm{~nm}$ was used to suppress spurious shoulders on the interstitial size distribution peak for the thin foil ion irradiation that would otherwise be caused by the non-uniformity of defect evolution at different depths. The mean interstitial cluster radius and mean vacancy cluster radius are $8.1 \mathrm{~nm}$ and $0.17 \mathrm{~nm}$, respectively, for the base neutron irradiation, and $5.9 \mathrm{~nm}$ and $0.18 \mathrm{~nm}$ for the thin foil ion irradiation.

Since defect evolution driven by ion irradiation in a thin foil depends on the depth, it is interesting to examine whether the neutron-induced damage can be better matched by the ion-induced damage at a particular depth than that averaged over the foil thickness. Here we examine the foil center where the free surface sink effect has the least influence (compared with other depth positions).

The open symbols in Fig. 7 represent the best matching of the defect concentration/density evolution at the center (depth) of a $60 \mathrm{~nm}$ foil at a dose rate of $3 \times 10^{-4} \mathrm{dpa} \mathrm{s}^{-1}$ to the same neutron damage 
as in Figs. 5 and 6. In this case, computation was still performed with full spatial dependence, including spatial grids near the free surfaces, but only the concentrations/densities at the central grid were used to match the defect evolution and determine the temperature shift. A slightly higher temperature $\left(200{ }^{\circ} \mathrm{C}\right)$ than that $\left(190^{\circ} \mathrm{C}\right)$ used above in the case of the depth-averaged ion damage, was required here to achieve the best matching. This is because, by only considering the foil center for the matching, the dose rate whose effect is to be overcome by the matching is virtually increased. Comparing Figs. 5 and 7, one can notice that the orders of magnitude of the total vacancy and total interstitial concentrations at the foil center in this case resemble those in the neutron damage more than the averaged concentrations across the foil thickness in the previous case, while in both cases the visible loop density is matched to the neutron irradiation quite well. The size distribution curves at $1 \mathrm{dpa}$ in this case are presented in Fig. 8 by the thin solid (green) lines. Although the interstitial cluster size distribution curve is sharper here, the mean interstitial cluster radius and mean vacancy cluster radius, $6.9 \mathrm{~nm}$ and $0.18 \mathrm{~nm}$ here, are in better agreement with the neutron irradiation than the previous case (Fig. 6).

The gap in Fig. 7 between the total vacancy (open circles) and total interstitial (open rhombuses) concentration trajectories suggests that the surface sink effect still has a clear influence on the matching of the defect evolution at the center of the ion irradiated $60 \mathrm{~nm}$ foil to the neutron damage, at least up to 1 dpa. Since the surface effect depends on the foil thickness, it is interesting to examine whether and, if yes, how much a thicker foil can improve the matching of the ion damage and the neutron damage.

The filled symbols in Fig. 7 represent the best matching of the defect concentration/density evolution at the center of a $180 \mathrm{~nm}$ foil at a dose rate of $3 \times 10^{-4} \mathrm{dpa} \mathrm{s}^{-1}$ to the same neutron damage as in Figs. 5 and 6. In this calculation, we used the same defect production probabilities as listed in Table S1 for the $60 \mathrm{~nm}$ foil, neglecting the minor variation in the PKA spectrum associated with the thickness change. It is also noted that about $30 \%$ of $1 \mathrm{MeV} \mathrm{Kr}$ ions injected into the $180 \mathrm{~nm}$ Mo foil are stopped inside the foil, according to SRIM. The effect of the stopped ions on the defect evolution is not considered here. However, among the $30 \%$ stopped ions, only $6 \%$ are stopped between the depths of $70 \mathrm{~nm}$ and 110 
$\mathrm{nm}$, which are $20 \mathrm{~nm}$ below and above the foil center. It is likely that the stopped ions do not significantly affect the defect evolution near the foil center that is concerned here. Comparing the results for the centers of the $60 \mathrm{~nm}$ (open symbols) and the $180 \mathrm{~nm}$ (filled symbols) foils in Fig. 7, one can find that the thicker foil leads to a narrower gap between the total vacancy and total interstitial concentration trajectories and a better agreement of the magnitudes of these quantities with those in the neutron irradiation. The size distribution curves at $1 \mathrm{dpa}$ for the center of the $180 \mathrm{~nm}$ foil are included in Fig. 8 as dashed lines. The position and width of the interstitial size distribution peak of the $180 \mathrm{~nm}$ foil agrees with the neutron damage better than that of the $60 \mathrm{~nm}$ foil. The mean interstitial-cluster and vacancy-cluster radii are 8.4 $\mathrm{nm}$ and $0.19 \mathrm{~nm}$, respectively, close to the $8.1 \mathrm{~nm}$ and $0.17 \mathrm{~nm}$ for the neutron damage.

In the end, we point out that some previous experiments ${ }^{23}$ have suggested that the defect evolution in irradiated materials may, beyond some dose level, deviate from the random nucleation and growth type of kinetics as described by the mean field rate theory, and undergo highly localized processes such as spatial correlation and rafting of interstitial loops. This has not been considered in the present study, nor in most existing rate theory or cluster dynamics studies.

\section{Conclusions}

To summarize, we have explored the possibility of matching defect evolution paths under varying conditions in thin foil heavy ion irradiation and bulk neutron irradiation of molybdenum, within a low dose and low temperature regime. Despite the high level of complexity in the underlying physics including intra-cascade defect cluster production, defect cluster mobility etc., it is found that a single temperature shift is adequate, in either the thin foil ion irradiation or the bulk neutron irradiation, to compensate for the effect of a changing dose rate and to match the time-dependent defect evolution (in terms of both defect concentrations/densities and size distribution) under dose rates differing by orders of magnitude. When using the thin foil ion irradiation to match the bulk neutron irradiation damage, surface sink effect associated with thin foils creates a disparity in the magnitudes of the total vacancy and total interstitial concentrations in the thin foil ion irradiation which fall above and below, respectively, the 
point defect concentrations in the bulk neutron irradiation, even when the visible interstitial loop density is matched well to that in the neutron irradiation by the temperature shift strategy. The agreement with the neutron irradiation can, however, be improved by choosing to match the defect evolution at the center of the foil only, instead of the averaged evolution across the foil thickness, or by using a thicker foil.

The reason for the success of the temperature shift strategy reported here, and the applicability and effectiveness of the temperature shift strategy at higher temperatures are currently under investigation.

\section{Acknowledgments}

We thank Gary Was for useful discussions. Xu and Wirth acknowledge financial support from the U.S. Department of Energy, Office of Nuclear Energy's Nuclear Energy University Program (NEUP), for the Integrated Research Project (IRP) on High Fidelity Ion Beam Simulation of High Dose Neutron Irradiation. VanCoevering acknowledges the NEUP graduate fellowship.

\section{References}

1. G. S. Was, Fundamentals of Radiation Materials Science: Metals and Alloys, Springer: New York, 2007.

2. D. R. Olander, Fundamental Aspects of Nuclear Reactor Fuel Elements, ERDA Publication, 1976.

3. S. J. Zinkle and G. S. Was, Acta Mater. 61, 735 (2013).

4. Y. Guerin, G. S. Was and S. J. Zinkle, MRS Bull. 34, 10 (2009).

5. T. R. Allen, J. T. Busby, R. L. Klueh, S. A. Maloy, and M. B. Toloczko, JOM 60, 15 (2008).

6. S. J. Zinkle and J. T. Busby, Mater. Today 12, 12 (2009).

7. M. J. Demkowicz, P. Bellon, and B. D. Wirth, MRS Bull. 35, 992 (2010).

8. G. S. Was et al., Scripta Mater. 88, 33 (2014).

9. M. A. Kirk et al., Microsc. Res. Techq. 72, 182 (2009). 
10. R. C. Birtcher, M. A. Kirk, K. Furuya, G. R. Lumpkin, and M. O. Ruault, J. Mat. Res. 20, 1654 (2005).

11. L. K. Mansur, J. Nucl. Mat. 216, 97 (1994).

12. L. K. Mansur, J. Nucl. Mat. 78, 156 (1978).

13. J. F. Ziegler, J. P. Biersack, and U. Littmark, The Stopping and Range of Ions in Matter, Pergamon: New York, 1984.

14. D. H. Xu et al., Acta Mater. 60, 4286 (2012).

15. T. R. Waite, Phys. Rev. 107, 463 (1957).

16. C. J. Ortiz and M. J. Caturla, Phys. Rev. B 75, 184101 (2007).

17. S. I. Golubov, A. M. Ovcharenko, A. V. Barashev and B. N. Singh, Phil. Mag. A 81, 643 (2001).

18. P. M. Derlet, D. Nguyen-Manh and S. L. Dudarev, Phys. Rev. B 76, 054107 (2007).

19. See supplementary material at XXX for Tables S1-S3.

20. A. P. Selby, D. H. Xu, N. Juslin, N. A. Capps, and B. D. Wirth, J. Nucl. Mat. 437, 19 (2013).

21. M. M. Li et al., Phil. Mag. 92, 2048 (2012).

22. J. Marian, unpublished research. For The SPECTER program, see http://www.oecdnea.org/tools/abstract/detail/psr-0263.

23. M. M. Li, M. Eldrup, T. S. Byun, N. Hashimoto, L. L. Snead, and S. J. Zinkle, J. Nucl. Mat. 376, 11 (2008).

24. M. J. Caturla, N. Soneda, E. Alonso, B. D. Wirth, T. D. de la Rubia, and J. M. Perlado, J. Nucl. Mat. 276, 13 (2000).

\section{Figure Captions}

Figure 1. (color online) Defect concentration/density evolution in a $60 \mathrm{~nm}$ Mo foil under $1 \mathrm{MeV} \mathrm{Kr}$ ion irradiation at varying dose rates and temperatures.

Figure 2. (color online) Size distribution curves for the ion irradiation conditions in Fig. 1(b) at 1 dpa. 
Figure 3. (color online) Defect concentration/density evolution in bulk Mo under HFIR-relevant neutron irradiation at varying dose rates and temperatures. Note that the higher dose rate is artificial, as detailed in the text.

Figure 4. (color online) Size distribution curves for the neutron irradiation conditions in Fig. 3(b) at 1 dpa. Figure 5. (color online) Matching defect evolution in Mo thin $(60 \mathrm{~nm})$ foil ion irradiation and Mo bulk neutron irradiation.

Figure 6. (color online) Size distribution curves for the ion and neutron irradiation conditions in Fig. 5 at 1 dpa.

Figure 7. (color online) Matching defect evolution at the center of a $60 \mathrm{~nm}$ (open symbols) and a $180 \mathrm{~nm}$ (filled symbols) Mo thin foil under ion irradiation to Mo bulk neutron irradiation.

Figure 8. (color online) Size distribution curves for the ion and neutron irradiation conditions in Fig. 7 at 1 dpa. 

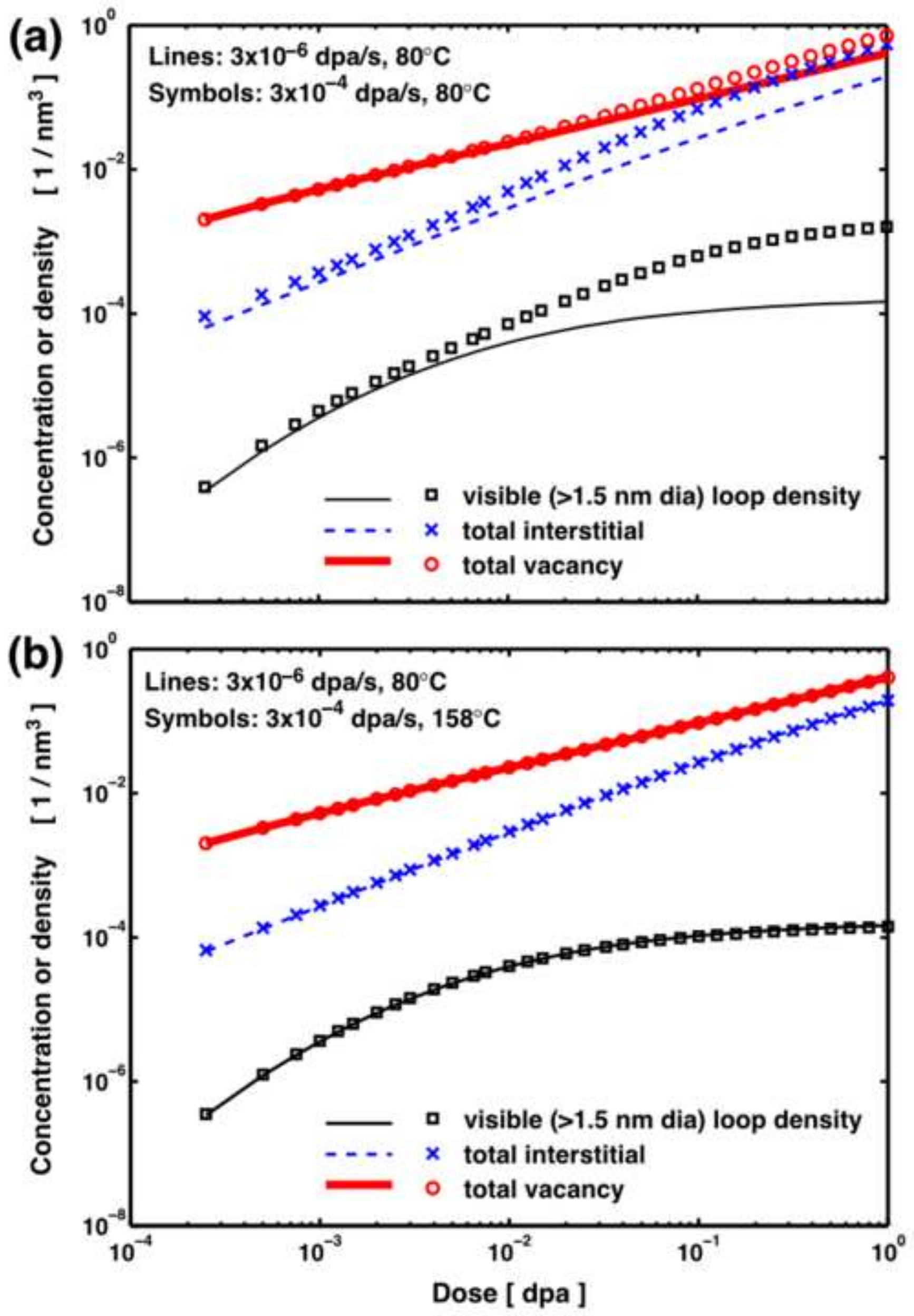


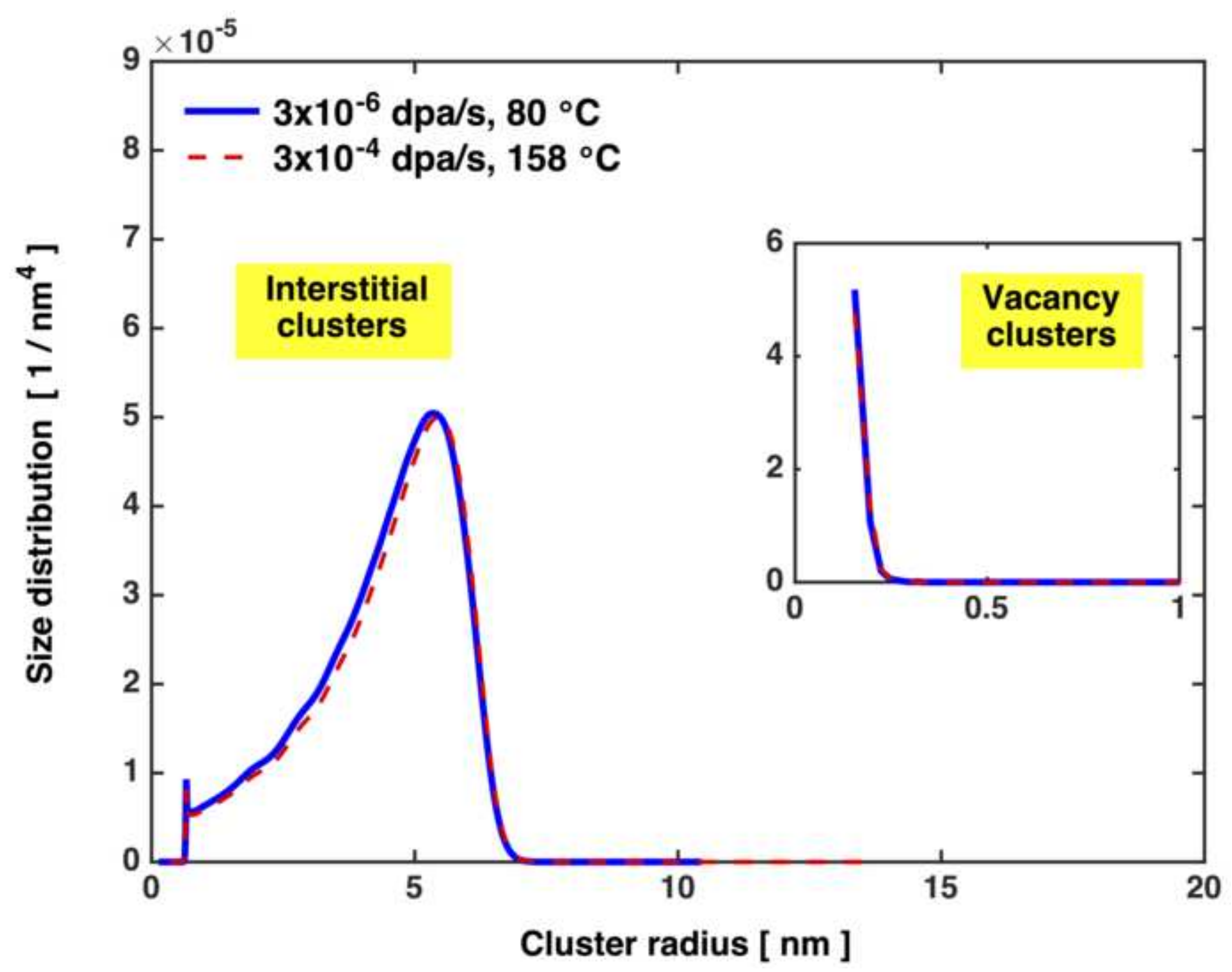



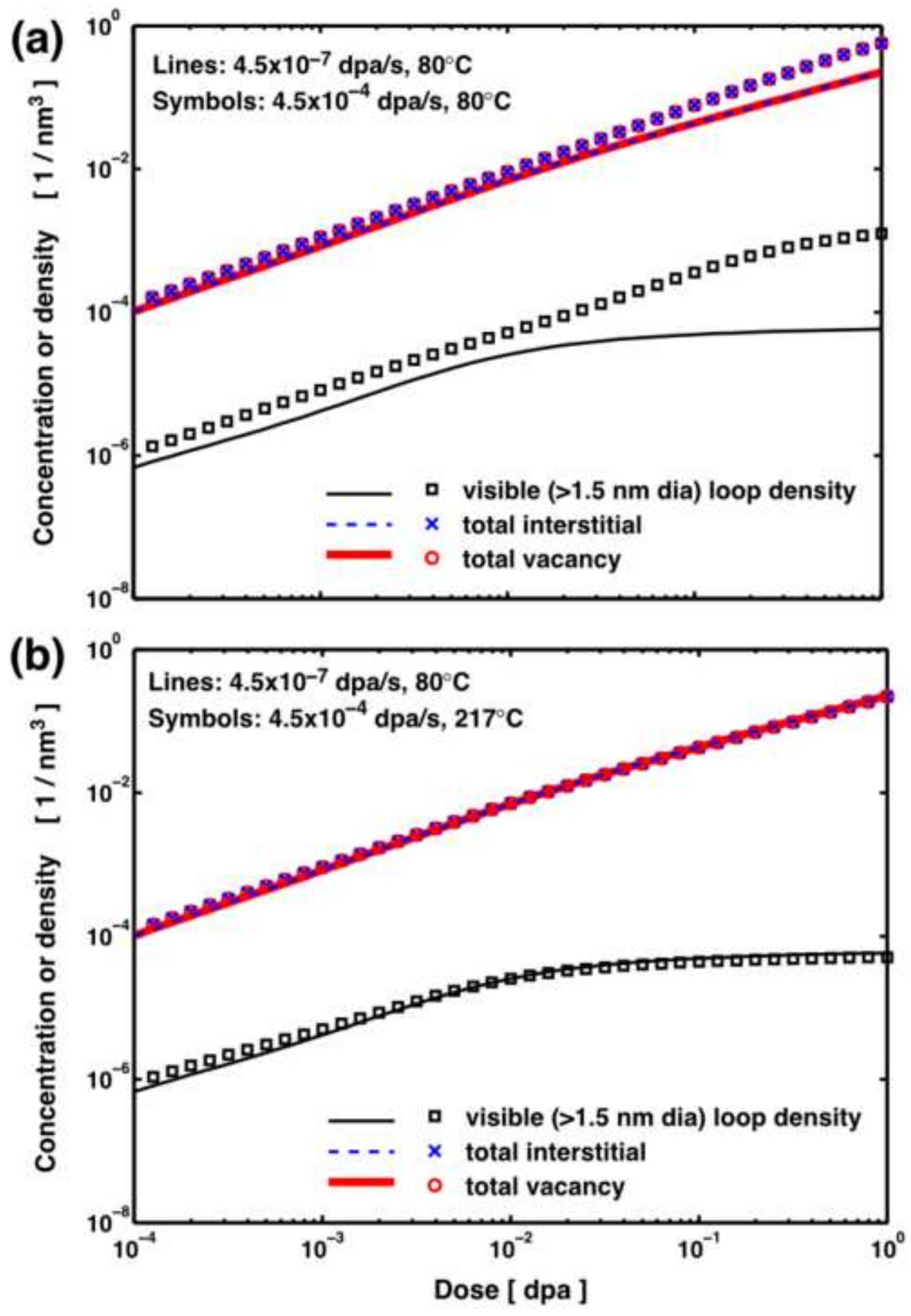


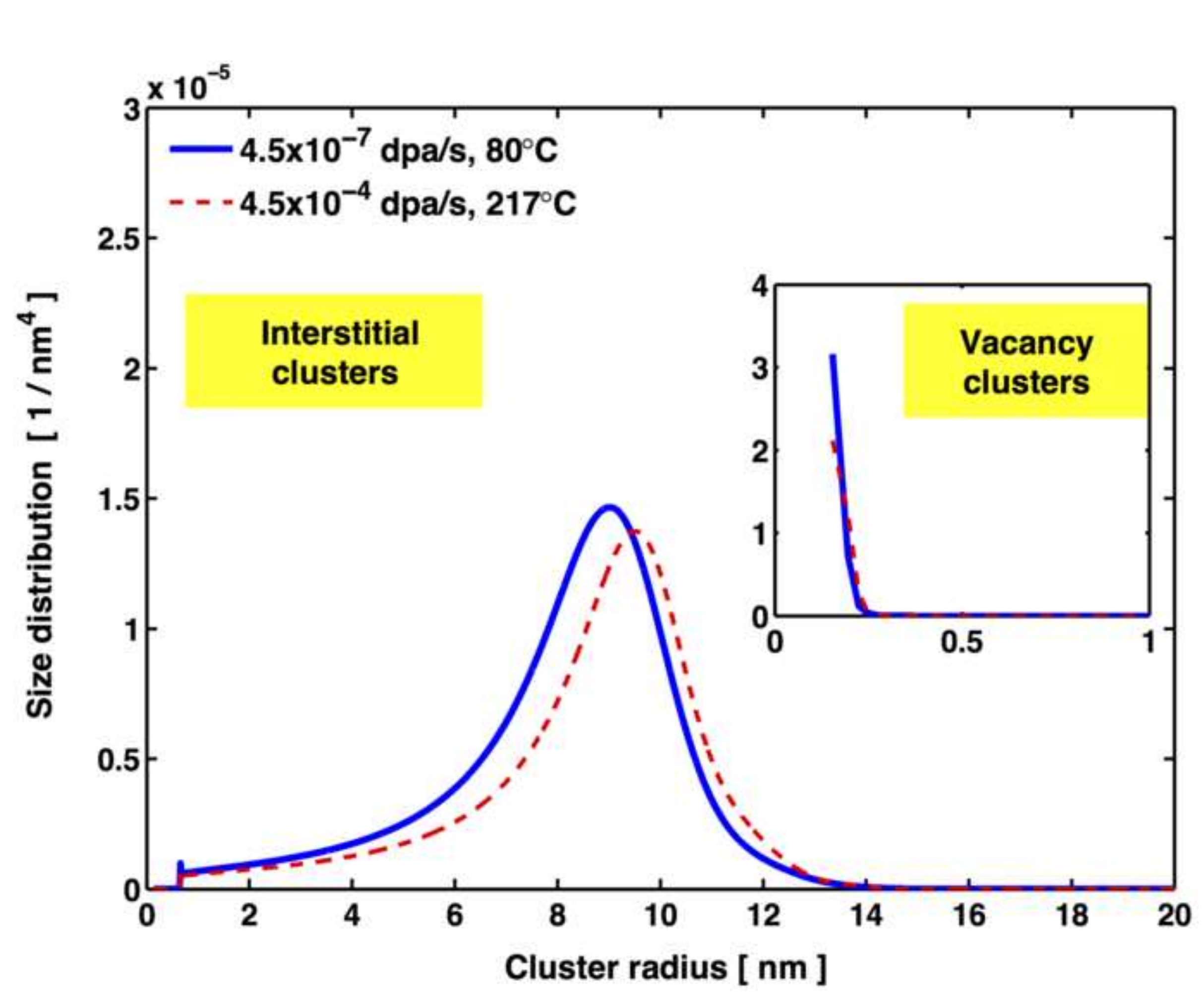




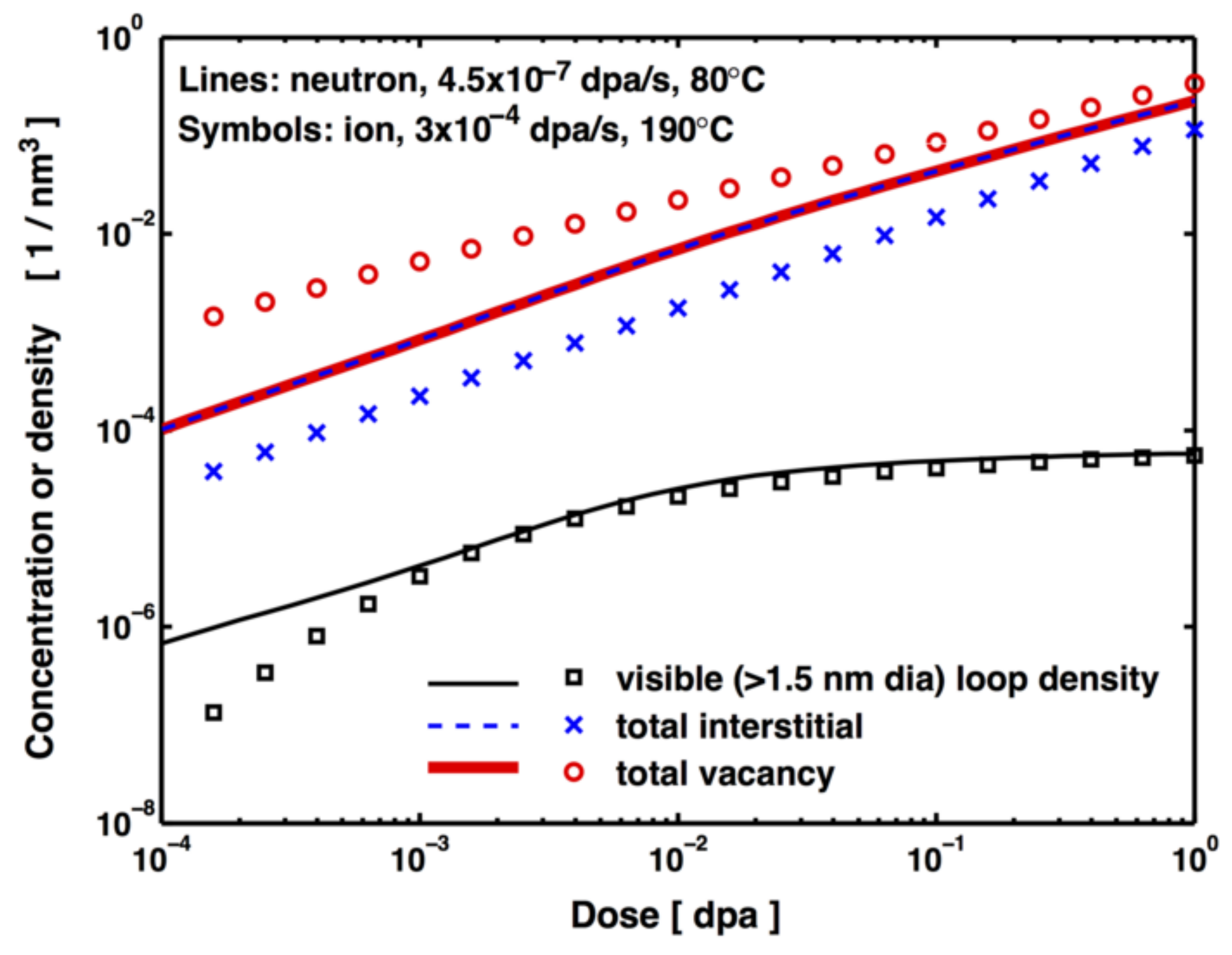




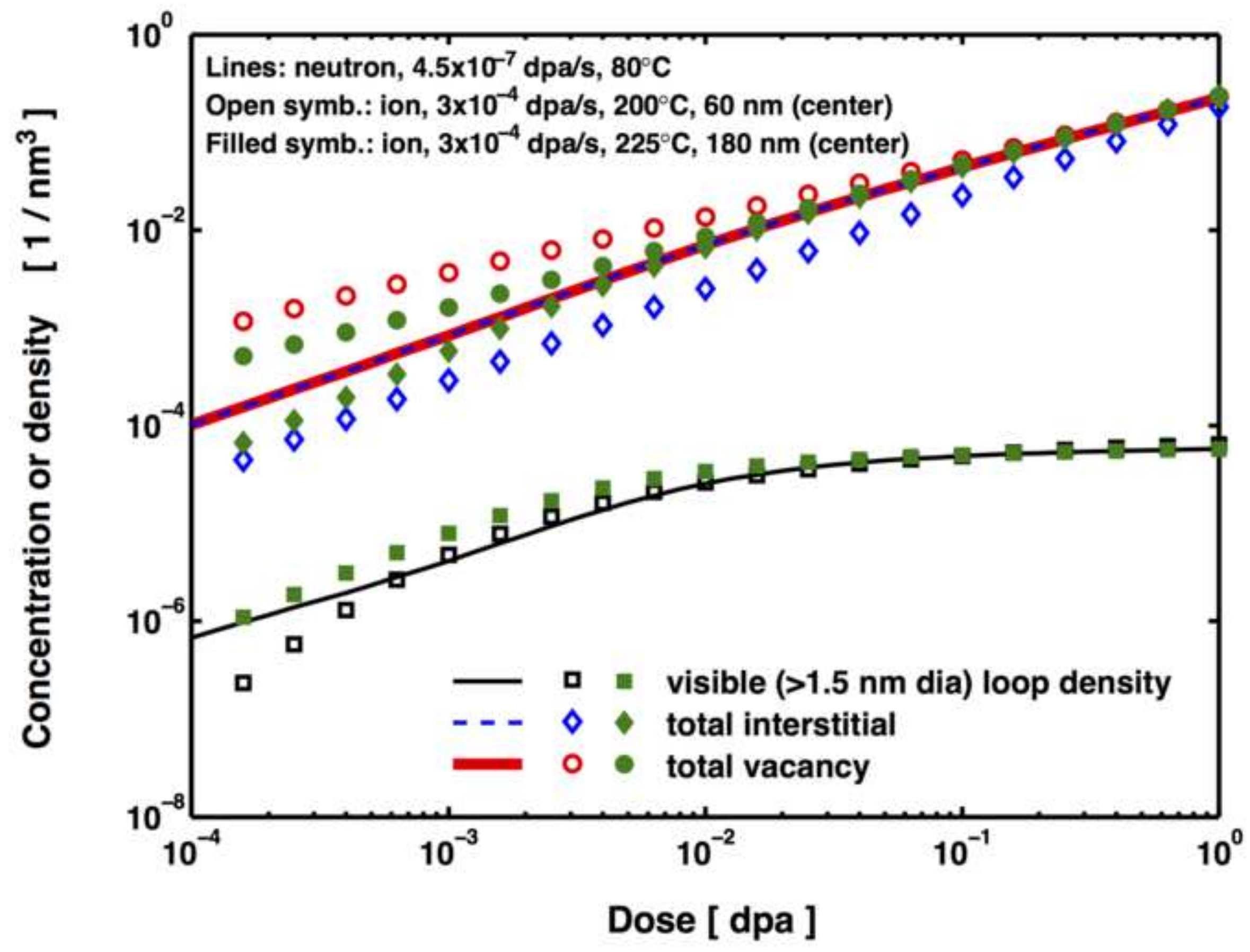




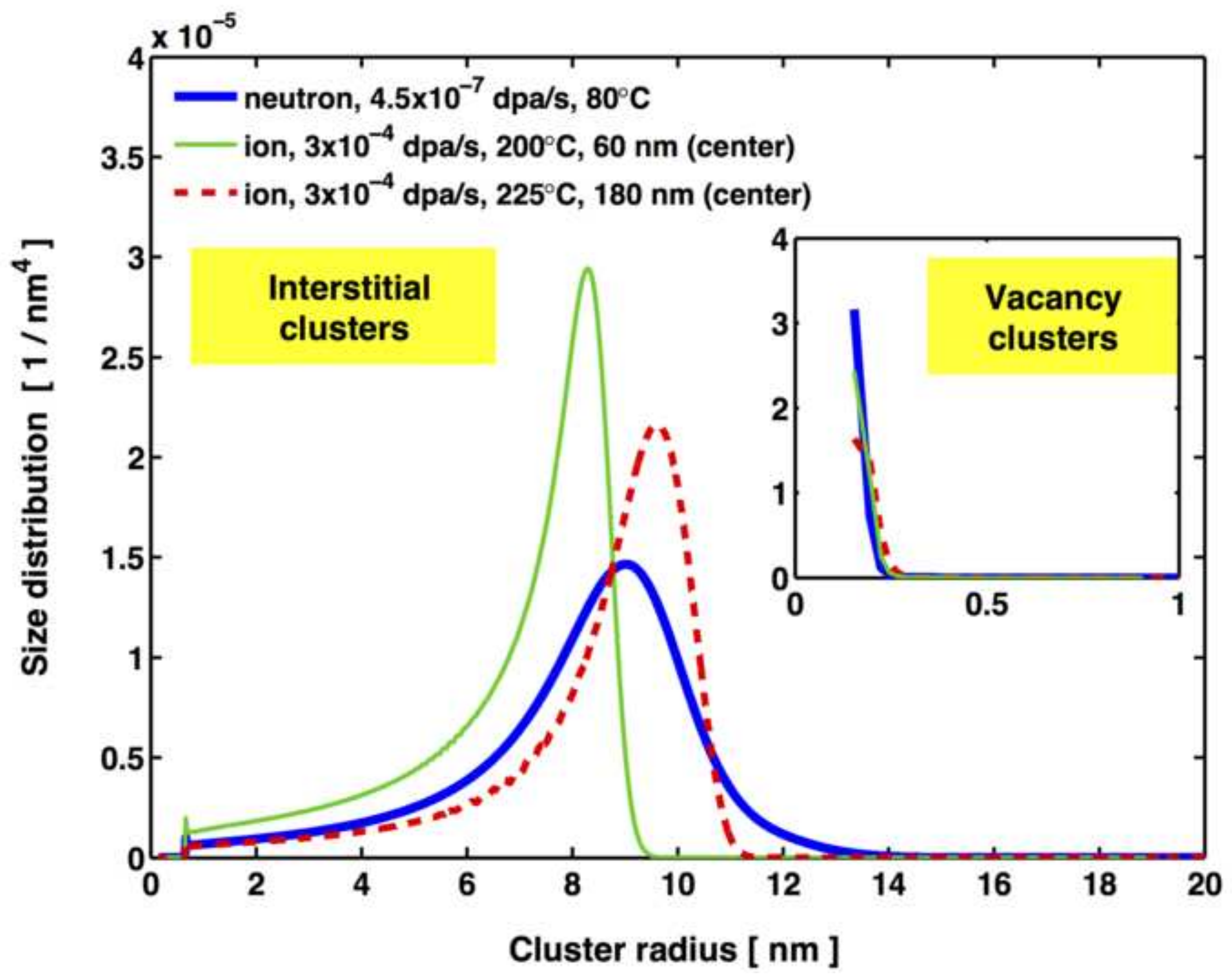

